\title{
El modelo argumentativo de Toulmin y la eficacia de titulación ${ }^{1}$
}

\section{The Toulmin Model of Argumentation and Graduation Rates}

\author{
Dení Stincer Gómez (*) dstincerg@gmail.com \\ Bertha Blum Grynberg (*) bblum@gmail.com
}

(*) Universidad Nacional Autónoma de México

(Recibido: 31 de marzo de 2016; Aceptado para su publicación: 28 de junio de 2016)

Cómo citar: Stincer, D. y Blum, B. (2017). El modelo argumentativo de Toulmin y la eficacia de titulación. Revista Electrónica de Investigación Educativa, 19(4), 9-19. https://doi.org/10.24320/redie.2017.19.4.1331

\section{Resumen}

El presente artículo aborda una posible solución a la baja eficiencia de titulación de los estudiantes de nivel superior, específicamente de posgrado, pues en las Instituciones de Educación Superior (IES) mexicanas de cada 10 egresados sólo 2.9 se titulan. Las causas son diversas y destacan la poca constancia de los estudiantes o la ineficacia institucional para apoyar esta tarea; la tesis es un discurso argumentativo que requiere un acompañamiento intelectual sistemático, apoyado en modelos de esta naturaleza. Se eligió el modelo heurístico argumentativo de Toulmin, que permite organizar las representaciones mentales en un inicio desorganizadas y confusas alrededor de un tema de investigación. La experiencia con cuatro generaciones en una residencia del programa de maestría de la Facultad de Psicología de la Universidad Nacional Autónoma de México (UNAM) y el análisis del comportamiento de las titulaciones desde 2012 al 2015 constatan que el uso del modelo resulta promisorio para incrementar la eficiencia de titulación.

Palabras clave: Modelo argumentivo, Toulmin, eficiencia, tesis, posgrado.

\section{Abstract}

This paper discusses a possible solution to low graduation rates in higher education, and particularly postgraduate students, given that for every 10 students that complete their course programs in Mexican higher education institutions, only 2.9 graduate. There are a number of reasons for this, notably student inconsistency and a lack of support from institutions. A thesis is an argumentative discourse that requires systematic intellectual support based on models of this nature. Toulmin's heuristic model of argumentation was chosen in order to organize, around a subject of research, mental representations that are, at the outset, disorganized and vague. The experience with four classes in a residency of the master's program in the School of Psychology of the National Autonomous University of Mexico (UNAM), and analysis of student graduation from 2012 to 2015, produce a promising model for increasing graduation rates.

\footnotetext{
${ }^{1}$ Este trabajo es producto del proyecto de investigación financiado por el Programa para el Desarrollo Profesional Docente en Educación superior (PRODEP) denominado "Eficacia del modelo argumentativo de Toulmin para la elaboración de tesis de grado y el incremento en la eficiencia de titulación en programas de posgrado".
} 
Keywords: Argumentative model, Toulmin, efficiency degree, thesis.

\section{Introducción}

La tesis de grado es una exigencia académica en muchas de las Instituciones de Educación Superior (IES) y un requisito para la acreditación de un grado en diversos países. Se interpreta como el trabajo que garantiza que los egresados cuentan con los conocimientos y las competencias para ejercer la profesión e investigar. Sin embargo, los índices de titulación muestran que "hacer la tesis" es una quimera. Por su alta exigencia intelectual un número importante de estudiantes no la concluye. De acuerdo con López, Albíter y Ramírez (2008), que citan datos provenientes de la Asociación Nacional de Universidades e Instituciones de Educación Superior (ANUIES), el promedio nacional de eficiencia terminal en México, considerando titulación, en el nivel de Licenciatura y en IES cuyo requisito es éste, es de 39\%, pero según Díaz de Cossío es de $12 \%$. Sea una u otra la cifra, menos del $50 \%$ de los estudiantes obtiene el grado, por lo que la eficiencia de titulación es baja.

La eficiencia de titulación consiste en la cantidad de estudiantes que cumplen con los créditos del programa en el que están inscritos y se titulan; es un indicador de éxito institucional (Barrera y León, 2012), sin considerar el tiempo que tarda el estudiante entre el cumplimiento total de los créditos y la obtención del título. La eficiencia de titulación tendría, así, niveles o grados. Existe mayor eficiencia si los estudiantes se titulan con la mayor proximidad posible al cumplimiento total de los créditos (con suficiente y probada calidad académica también) que si transcurre mucho tiempo. Estos tiempos no se han consensuado aún y las cifras institucionales son confusas, pues reportan un número de titulados por año escolar sin precisar el tiempo transcurrido entre un evento y otro. Aun así, las cifras son bajas. En la agenda Estadística UNAM 2015 (en el nivel de posgrado), los datos indican que de 9,500 estudiantes, 3,057 se titulan, ${ }^{2}$ casi una tercera parte. En Licenciatura, del $100 \%$ de estudiantes $(21,734) 66.8 \%$ opta por otras alternativas de titulación antes que por la tesis, sólo una tercera parte resuelve hacerla.

Fernández, Mendoza, Rodríguez y López (2013) mencionan diversos factores que inciden en los bajos índices de titulación. Constatan en sus investigaciones lo que también refieren Luna (2011), Medina (2008), Rodríguez (2006) y Villagra y Casa (2010). Los bajos índices de titulación se asocian a la alta carga de trabajo que implica, escasa comunicación con los asesores y dificultades de los estudiantes para la escritura. Los autores plantean que los estudiantes tienen poca certeza sobre lo que desean investigar. Desde el punto de vista institucional consideran que hay una inadecuada planeación del proceso, la asignación de tutores es arbitraria, existe una desvinculación entre los seminarios de investigación y los trabajos de los estudiantes y, por último, son inexistentes o insuficientes los foros en los que el estudiante tiene la oportunidad de exponer sus ideas y trabajos frente a un auditorio.

López, Salvo y García (1989) señalan que los estudiantes tienen fuertes carencias metodológicas y son inexpertos en el campo de la investigación, tienen dificultades para integrar los conocimientos adquiridos en un proyecto personal, carecen de habilidades para la comprensión de lectura y la redacción de textos, para sintetizar ideas centrales, hacer críticas fundamentadas y, de forma general, copian textualmente como estrategia.

Otra de las causas está relacionada con una cuestión "de fondo", de naturaleza más psicológica. Hacer una tesis de grado implica involucrarse en un proceso epistemológico, inmiscuirse en un diálogo argumentativo y en actividades experimentales o probatorias. El producto es un discurso donde debe probarse una propuesta de conocimiento, un supuesto o hipótesis, hacer claras las evidencias, justificar la plausibilidad de las hipótesis apelando a los mejores argumentos, diseñar un método y tomar en cuenta los contraargumentos y críticas. Todo ello debe, además, exponerse en forma oral y escrita, lo que lo convierte en un proceso intelectual y afectivamente complejo.

\footnotetext{
${ }^{2}$ En este dato no se precisa el año en el que los estudiantes que se titularon culminaron sus créditos, por lo que sí se toma en cuenta la cercanía de la titulación con la culminación de los créditos, se desconoce el grado real de eficiencia de titulación.
} 
Los componentes antes mencionados forman parte de la estructura de un buen argumento y la tesis es un discurso argumentativo. Este trabajo propone retomar un modelo que proviene de la filosofía de la ciencia (de Stephen Toulmin) y a través de él proveer de acompañamiento intelectual a los estudiantes para la construcción de su tesis.

\subsection{El modelo argumentativo de Toulmin}

Stephen Toulmin fue un matemático y filósofo de la ciencia, estudioso del discurso argumentativo que condensó en un modelo los componentes básicos de un buen argumento, no sólo los de orden lógico, sino los de orden social y pragmático (Van Eemeren y Grootendorst, 2004) y los persuasivos considerados por Perelman y Olbrecht (2006). Estos componentes son: la hipótesis, las evidencias, las justificaciones, los respaldos o fundamentos y los refutadores que, a su vez, dan lugar a los calificadores modales o términos moderadores (ver figura 1).

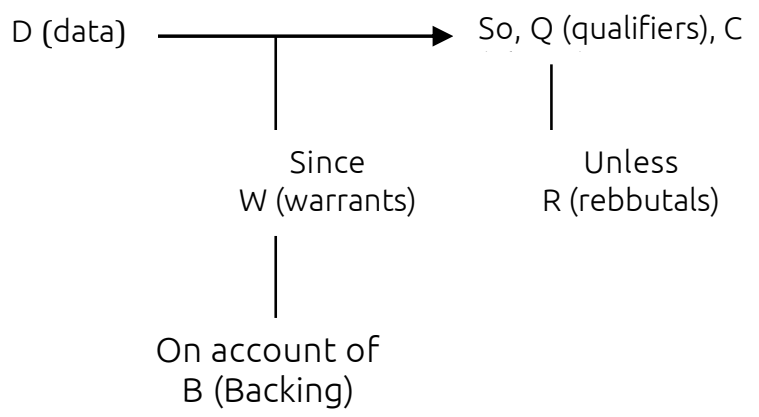

Figura 1. Esquema del modelo de Toulmin (1958)

La estructura del modelo da cuenta de: 1) los componentes que son necesarios en un buen argumento, y 2) el orden en el que deben ir estos componentes dentro del discurso. El modelo debe comprenderse de la siguiente manera. A partir de un conjunto de hechos, evidencias y datos (los primeros componentes, data), nuestra mente puede realizar una inferencia (segundo elemento del modelo, claim). A esa inferencia, dentro de los procesos metodológicos de la investigación, también se le conoce como hipótesis o supuesto. Esa inferencia es una construcción mental de "algo" que revelan los datos. Puede concebirse como una abstracción de cualidades intrínsecas a ellos, de relaciones implícitas, ocultas o difíciles de percibir, de manera que debemos comprometernos a probarlas. Realizada la inferencia es necesario justificarla, dar las razones que permiten considerar que es cierta. En el tercer elemento del modelo, warrants (garantías), es el momento en el que se hacen explícitas las razones, reglas y principios que permiten percibir a la hipótesis como un enunciado cierto o plausible. Dentro de la argumentación, esta tarea es una de las más arduas porque no sólo exige buscar evidencias sino hacer explícitas las razones teóricas que explican el contenido y las relaciones involucrados en la inferencia inicial.

Es importante resaltar que en la tarea de justificar, las razones a las que acudimos provienen de las teorías que nos "gustan" o compartimos con nuestra comunidad. Al no existir en la teoría, estas razones deben provenir del sujeto de investigación, por eso es el momento más complejo -y el más creativo- para el que el estudiante en formación, en general, no está preparado. Lo recomendable es que desde el inicio busque apoyo en los teóricos de su preferencia, para que después pueda formular argumentos propios.

El cuarto elemento son los respaldos (backing); una vez explícitas las razones por las cuales la hipótesis puede ser cierta o plausible es necesario mostrar los autores que sustentan los argumentos a los que apelamos.

Uno de los componentes más importantes son los refutadores (rebbutals), el quinto elemento. Son las hipótesis conocidas en la metodología como hipótesis nula o alterna, aquellos enunciados (o inferencias) 
que contradicen, desafían o pueden coexistir con lo que inferimos. De las mismas evidencias puede desprenderse otra inferencia o existir argumentos que la nieguen, y hay que tomarlos en cuenta porque pueden ser epistemológicamente relevantes. La refinación de una hipótesis o un supuesto depende de cuánto se haya considerado y resista el punto antagónico. Los absolutismos de las primeras inferencias van desapareciendo en la medida que se consideran los puntos de vista contrarios. De estas últimas consideraciones aparece el sexto elemento, al que Toulmin llama cualificadores modales, términos que se incluyen en la inferencia, y cuya función es moderar "los absolutismos" considerando los márgenes de error y los posibles contraejemplos. No es lo mismo decir "100\% de los fumadores enferma de cáncer", que "90\% de los fumadores enferma de cáncer", la última afirmación considera los contraejemplos o a aquellas personas que fumaron muchos años y no padecieron la enfermedad.

\subsection{El modelo de Toulmin como heurístico mental para la elaboración de tesis}

Nuestra propuesta es que el modelo de Toulmin funcione como un heurístico. Los heurísticos, desde las psicologías cognitivas, son considerados mecanismos, instrucciones o reglas mentales que permiten solucionar problemas específicos y hacen más eficientes los procesos cognitivos, sobre todo aquellos que parecen ser computacionalmente costosos. No necesariamente llevan a soluciones correctas o son garantías de obtener la solución, pero es como racionalmente actuamos (Eraña, 2003): "son sistemas de instrucciones que nos permiten solucionar un problema, no de manera exacta ni en todos los casos, pero sí de manera que no sea necesaria una computación excesiva de los recursos cognitivos que tenemos disponibles". Eraña (2003) considera que la competencia racional está constituida por reglas heurísticas. Otro aporte interesante es el de Pérez (1990), quien los considera mecanismos por los que reducimos la incertidumbre que produce nuestra limitación para enfrentarnos a la complejidad de estímulos ambientales. Son de naturaleza psicológica y lingüística y no matemática, basados en la actuación humana frente a la resolución de problemas (De Gracia y Castelló, 2002).

De acuerdo con estos supuestos, los heurísticos son mecanismos mentales encriptados por la experiencia y las relaciones entre el hombre y su medio. Instrucciones que han quedado impresas en la mente (o en los circuitos neuronales), cuya función permite solucionar algunos problemas. Cada proceso cognitivo parece tener sus heurísticos y sus reglas. Suponiendo que la argumentación es un proceso cognitivo podemos inferir que el modelo de Toulmin es el heurístico para ello, pues contiene las instrucciones y las reglas para argumentar.

La mente recibe los datos, los procesa con sus esquemas previos e infiere o construye un enunciado que después tendrá que probar con evidencias y explicar con argumentos, deberá buscar apoyos, respaldos y considerar los refutadores. Toulmin hizo explícita la estructura del heurístico de la argumentación. Hizo externo lo que ocurre en el plano interno cuando estamos en esta empresa epistemológica. Estando ahora el modelo interno en el plano externo se convierte en un andamio vigotskiano que requerimos para que los estudiantes hagan uso de esta estructura en su plano interno.

\subsection{Didáctica con el modelo de Toulmin}

El uso del modelo de Toulmin para elaborar la tesis de grado parte de dos supuestos fundamentales: 1) la tesis es un discurso argumentativo y 2) a los estudiantes, de forma general, les atrae un tema de investigación. Esto significa que sienten curiosidad por algunos datos que les resultan interesantes y ya empiezan a tener o tienen alguna inferencia.

Accediendo el estudiante al tema de su interés y comprendidos los componentes del modelo de Toulmin comienza el trabajo de ubicar las distintas representaciones acerca del tema en el lugar del modelo que corresponde y construir aquellas que faltan. En la tabla I aparecen los componentes del modelo y ellos van escribiendo en cada recuadro lo correspondiente. 
Tabla I. Formato para organizar las representaciones mentales de acuerdo con cada componente del modelo

\begin{tabular}{|c|c|}
\hline Evidencias & Hipótesis o supuesto \\
\hline \multicolumn{2}{|c|}{ Justificaciones } \\
\hline \multicolumn{2}{|c|}{ Fundamentos o respaldos } \\
\hline \multicolumn{2}{|c|}{ Refutadores } \\
\hline
\end{tabular}

Nota: La extensión de los recuadros varía según el texto escrito en ellos.

En la columna izquierda de la tabla se escriben los datos, hechos (evidencias) que son significativos para el estudiante, luego ocurre una acción: construir lingüísticamente la inferencia inicial de estos datos. Es la primera hipótesis, y por muy intuitiva y tosca que sea es la primera abstracción derivada de los datos.

Luego, tanto las evidencias como esta primera inferencia se someten a discusión con el grupo. Éste, al ser parte de un mismo programa educativo, criticará desde un similar bagaje teórico. Los compañeros son el filtro principal para la depuración lógica de las relaciones vistas entre las evidencias y la primera hipótesis del proponente. En esta fase del debate se intenta que la inferencia inicial muestre relaciones de implicación plausibles con las evidencias expuestas. Es decir, que $p$ (los datos) implique, en la mayor medida posible, $q$ (el supuesto o hipótesis).

En el terreno argumentativo esta situación de debate tiene definidos a sus personajes: un proponente y los oponentes. Estos últimos, al escuchar la inferencia del proponente y compartir algunas representaciones teóricas similares, tienden a ejercer una acción crítica revelando las aristas que el proponente no ve. Son los primeros impulsores de los contraejemplos y las hipótesis alternas, que posteriormente pueden hacer al proponente adoptar la actitud más relevante desde el punto de vista epistemológico: 1) incluir los elementos faltantes en su inferencia, completándola o 2) retractarse y asumir otra inferencia que le resultó más viable y congruente con sus evidencias. Esta situación de debate debe ser conducida por el moderador (el docente) quien deberá dar cauce positivo a toda suerte de falacias informales que puedan atentar con el buen discurrir de la discusión (Van Eemeren y Grootendorst, 2004).

El proceso de refinamiento de la inferencia o hipótesis debe someterse al mayor escrutinio posible, una hipótesis "bien afinada" es crucial para la delimitación de las tres partes más importantes de una tesis de grado: 1) la identificación de los contenidos del marco teórico, 2) el diseño del método y 3) la interpretación de los resultados. Es por ello que luego del escrutinio grupal el estudiante lo da a conocer al asesor asignado institucionalmente, quien por su trayectoria académica se supone aportará al refinamiento de las inferencias realizadas.

Una vez que la inferencia y las evidencias pasaron por el escrutinio público sigue la fase de justificar. Como explicamos en el apartado anterior, las justificaciones son las razones teóricas y/o empíricas que permiten admitir que la inferencia es correcta y entender por qué es cierta o plausible. Es el momento en el que los estudiantes deben recurrir a la teoría. La evidencia per se no es suficiente. También pueden darse cuenta que no dominan con solidez teoría alguna y es momento de profundizar en ello.

Para este trabajo mental se proporciona también información sobre los tipos de argumentos o la estructura de los buenos argumentos. Recurrimos nuevamente a la filosofía de la ciencia y la epistemología. En la tarea de argumentar o justificar podemos apelar a varias estructuras de argumentos. Se cuenta con los argumentos positivos o analíticos, los negativos (o contrajemplos), los de plausibilidad empírica, y los que apelan a la mejor explicación (o abductivos).

De acuerdo con la síntesis de Barceló (2012), los argumentos positivos son aquellas razones que apelan a las causas y razones dadas para "creer" en la inferencia que estamos sosteniendo, también apelan a las consecuencias. Son entonces las razones que apelan a las causas y efectos. Por ejemplo, si la hipótesis fuera que el cigarro provoca cáncer porque la evidencia muestra que la mayor parte de los fumadores enferman de cáncer, el hecho mismo de que las personas se enfermen no es suficiente para entender por qué el cigarro provoca cáncer. Un argumento positivo sería el que nos proporciona las razones de por qué el cigarro provoca cáncer. 
Los argumentos negativos son los contraejemplos o refutadores. Se trata de encontrar ejemplos o casos que nieguen la hipótesis, si son menos y sólo se cumplen en minoría y explican en menor medida la regularidad, entonces la inferencia es probablemente más cierta y plausible respecto al contraejemplo.

Los argumentos de plausibilidad empírica son aquellos que buscan más evidencias a favor de la hipótesis, pero evidencias obtenidas de forma sistemática, experimental o inductivamente. Mientras más fumadores enfermen de cáncer, la hipótesis de que el cigarro provoca cáncer se fortalece. Como podemos ver, no explica ni da razones para entender la hipótesis, pero sí la fortalece.

Los argumentos abductivos son muy interesantes, también analíticos, pero consisten en elegir dentro de todas las posibles razones causales aquella que mejor explique una conclusión. Los positivos apelan a las causas, los abductivos eligen todos los positivos existentes y apelan al que mejor explique el fenómeno.

Una vez conocidas y comprendidas estas estructuras argumentativas se invita al estudiante a justificar su inferencia apelando a estas estructuras, que son las más recurrentes en la actividad académico-científica. Identificadas las principales razones que contribuirían a entender la hipótesis se pasa al recuadro de los respaldos. Ahí se deben ubicar y escribir las fuentes que les proporcionaron esas razones a los proponentes.

Por último, se escriben los refutadores, inferencias o contraejemplos. En general, los proponentes retoman las hipótesis alternas que sus compañeros proporcionaron para preparar los argumentos que impidan que el contraejemplo sea más plausible que su inferencia. En todo el proceso la inferencia se va afinando para resistir las críticas más duras, hasta que puede ser compartida por los demás interesados en el tema.

Esta tercera parte, la de las justificaciones, los fundamentos y los refutadores, también pasa por el escrutinio del grupo y del asesor. El producto final es un recuadro con todas las representaciones mentales ordenadas en su sitio, listas para construirse en un discurso textual escrito.

\subsection{Proceso de transformación de las representaciones mentales organizadas de acuerdo con el modelo}

Una tesis de grado, de forma general, consta de cuatro partes sustanciales: 1) el planteamiento del problema que concluye con la pregunta de investigación y el supuesto o hipótesis, 2) el marco teórico, 3) el método, 4) los resultados, la discusión y las conclusiones. Cuando el estudiante tiene sus representaciones organizadas puede comenzar la escritura de cada parte.

En síntesis, el planteamiento del problema se beneficia del recuadro de las evidencias, que son narradas en forma de texto. Se solicita al estudiante que las narre desde su experiencia y luego retome otras investigaciones donde también las percibieron como un problema. Funciona muy bien limitar la narración a cinco cuartillas aproximadamente y concluir esta sección con la pregunta de investigación y el supuesto o hipótesis. La cual ya tienen formulada.

Posteriormente comienza una de las partes más delicadas, decidir el contenido del marco teórico. Para ello se toman como base la hipótesis y las justificaciones; por supuesto, el marco teórico se apoyará en los autores e investigaciones que se ubicaron en los respaldos. De manera que los tres elementos del recuadro son necesarios para su construcción. Siendo la hipótesis la idea que se desea defender o probar, el marco teórico debe contener una teorización sobre las categorías y las relaciones involucradas en ella. Observando las principales categorías de la hipótesis y sus relaciones, se desglosa el capitulado del marco teórico. 
Retomamos como ejemplo la siguiente hipótesis, que se refiere al estudio de un caso clínico: "Los atracones ${ }^{3}$ y el sobrepeso de Ninfa (una adolescente) son derivados de la falta de diferenciación con la madre, además de una reacción contra el rechazo materno y a fallas en la entrada de un tercero" (Carreño, 2015).

¿Qué se espera que contenga el marco teórico de esta tesis? Evidentemente el desarrollo de los siguientes temas: 1) los atracones y el sobrepeso en la adolescencia, 2) procesos de diferenciación, 3) rechazo materno y sus implicaciones en el estado físico de las hijas, 4) la ausencia paterna (a lo cual se está refiriendo cuando menciona las fallas en la entrada de un tercero). Por último, se deben integrar teóricamente las relaciones entre el fenómeno de los atracones, las fallas maternas y la ausencia de un padre. Así serían los capítulos del marco teórico, es innecesario abordar otros. Lo cual facilita al estudiante limitar el número de cuartillas a una extensión moderada.

Una vez construido el marco teórico se debe diseñar el método, que siempre se hace en función de evaluar (constatar/probar) la hipótesis o el supuesto. La inferencia sigue siendo el hilo conductor. Como ya está "afinada" corresponde invitar al estudiante a que diseñe el método desde la perspectiva metodológica con la que se identifica o considere más viable para probarla. Esto consiste en diseñar en función de las metodologías cualitativas, cuantitativas o mixtas. De forma general, no han hecho explícita su identificación con una de ellas y hay que rememorar las cualidades de ambas, sus principios, estrategias y técnicas para que logren esta identificación y delimiten con precisión su propio diseño.

Por último, el apartado más complejo es la sección de resultados, discusión y conclusiones, donde se retoman nuevamente la hipótesis y las justificaciones (que se enriquecieron con el marco teórico). En este trabajo se propone organizar los resultados eligiendo las evidencias que prueben cada sección de la hipótesis proporcionando los argumentos que la explican.

Retomando la hipótesis ejemplo, en primer lugar, se presentarán evidencias de los atracones y el sobrepeso de Ninfa, luego las evidencias de que esos atracones se deben a la falta de diferenciación con la madre. Esto implica que el investigador muestre los hechos donde podemos percibir que por esta falta de diferenciación con la madre, la hija se atraca. Después debe argumentar por qué es así y es ahí donde el sujeto investigador apela a las justificaciones. También debe dar evidencias de cómo la ausencia del padre influyó en el descuido de su cuerpo y argumentar desde la teoría por qué fue así. Es un trabajo arduo porque el estudiante comienza a relacionar evidencias con teoría, es decir, comienza a interpretar la evidencia.

\section{Método}

Para constatar la pertinencia del modelo sobre la eficiencia de titulación se acudió a un estudio descriptivo y transversal del comportamiento de la titulación en cuatro generaciones del Programa de Maestría y Doctorado en Psicología de la UNAM, específicamente de la Residencia en Psicoterapia para adolescentes.

La eficiencia de titulación es el número de estudiantes que se titula durante al menos tres semestres escolares después de concluidos los créditos, es decir, un año y medio. Comúnmente, la eficiencia de titulación es el número de estudiantes que una vez concluidos sus créditos se titula sin importar el año en que lo haga. La hipótesis en este trabajo es que el modelo promueve la titulación en tiempos muy próximos a la culminación de los créditos.

El modelo es parte de una de las materias del programa. Comienza formalmente en el año 2011 y ha atendido a cuatro generaciones (la quinta se encuentra en la mitad del proceso). Ocupa 6 horas semanales (2 teóricas y 4 prácticas) durante dos semestres escolares. La maestría tiene una duración de cuatro semestres y la materia en cuestión inicia en el tercer semestre. En ese momento los estudiantes ya

\footnotetext{
${ }^{3}$ Conducta compulsiva caracterizada por una forma incontrolada de comer que resulta en un aumento de peso (Cleveland Clinic, 2006).
} 
cuentan con un año de teoría y práctica profesional.

La inclusión de esta asignatura se debió a la baja titulación que presentaba la maestría en las generaciones que culminaron sus créditos en 2010 y 2011. Durante dos años consecutivos los números indicaban un gran problema (ver figura 2).

Generación 2010

Total 13 estudiantes

Año de titulación y cantidad de titulados

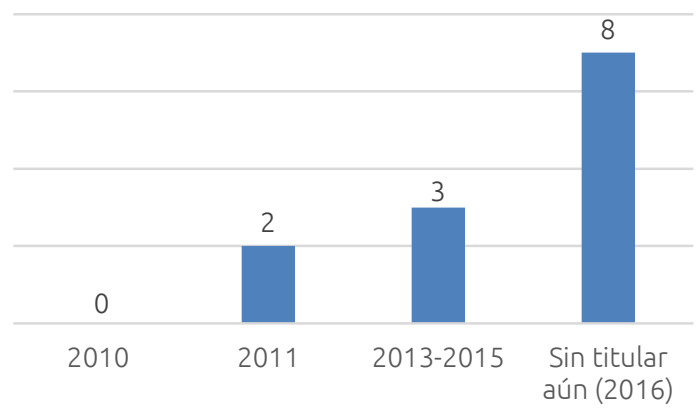

Generación 2011

Total 13 estudiantes

Año de titulación y cantidad de titulados

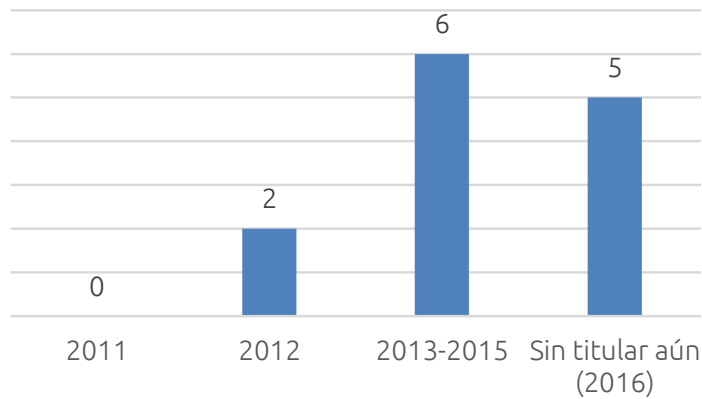

Figura 2. Estudiantes que culminaron sus créditos en los años 2010 y 2011, titulados, no titulados y año en el que obtienen el grado

Nota: Datos extraídos de la base de datos de la Coordinación de la Residencia en Psicoterapia para adolescentes, Facultad de Psicología, UNAM.

Como se puede observar en ambos casos, sólo dos estudiantes se titularon un año después de haber concluido los créditos. Hasta tres y cinco años después de culminar los créditos se tituló entre 20\% y 50\%, y hasta el 2016 (seis años después) el resto continúa sin titularse. De la generación 2010 el 62\% no se ha titulado, y de la generación 2011 sigue pendiente el $40 \%$.

Se inició la aplicación del modelo y para conocer el comportamiento de la titulación se construyó una base de datos con los nombres de los estudiantes, la fecha en que concluyeron sus créditos, la fecha de su examen de grado y obtención del título. Después de cuatro años y respecto a los dos años anteriores, la titulación incrementó significativamente, concentrándose la mayor parte de los exámenes de grado u obtención del título, en el 5o., 60. y 7o. semestre. Hay que tener en cuenta que concluyen sus créditos en el 4o. semestre (tabla II).

Tabla II. Datos de las generaciones que recibieron la materia con el modelo de Toulmin

\begin{tabular}{cccccccc}
\hline $\begin{array}{c}\text { Generación } \\
\text { en la que } \\
\text { comienza el } \\
\text { seminario }\end{array}$ & $\begin{array}{c}\text { Total de } \\
\text { alumnos }\end{array}$ & $\begin{array}{c}\text { Año que } \\
\text { culmina la } \\
\text { maestría }\end{array}$ & $\begin{array}{c}\text { Total de } \\
\text { alumnos } \\
\text { que culmi- } \\
\text { nan sus } \\
\text { créditos }\end{array}$ & $\begin{array}{c}\text { Alumnos } \\
\text { titulados } \\
\text { en el 5o. } \\
\text { semestre }\end{array}$ & $\begin{array}{c}\text { Alumnos } \\
\text { titulados } \\
\text { en el 6o. } \\
\text { y 7o. } \\
\text { semestre }\end{array}$ & $\begin{array}{c}\text { Alumnos } \\
\text { no } \\
\text { titulados a } \\
\text { la fecha } \\
(2016)\end{array}$ & $\begin{array}{c}\text { Eficiencia } \\
\text { de } \\
\text { titulación }\end{array}$ \\
\hline 2011 & 14 & 2012 & 14 & 0 & 7 & 7 & $50 \%$ \\
2012 & 15 & 2013 & 15 & 8 & 6 & 0 & $100 \%$ \\
2013 & 10 & 2014 & 10 & 2 & 4 & 4 & $60 \%$ \\
2014 & 14 & 2015 & 14 & 4 & 8 & 2 & $80 \%$ \\
\hline
\end{tabular}

\footnotetext{
${ }^{4}$ Estos 8 estudiantes se titularon durante enero y febrero del 2016, lo que corresponde al 6o. semestre (dos semestres posteriores al cumplimiento de sus créditos).
} 
En la figura 3 se observa el comportamiento de titulación entre las generaciones que no cursaron la materia con el modelo de Toulmin y los que sí.

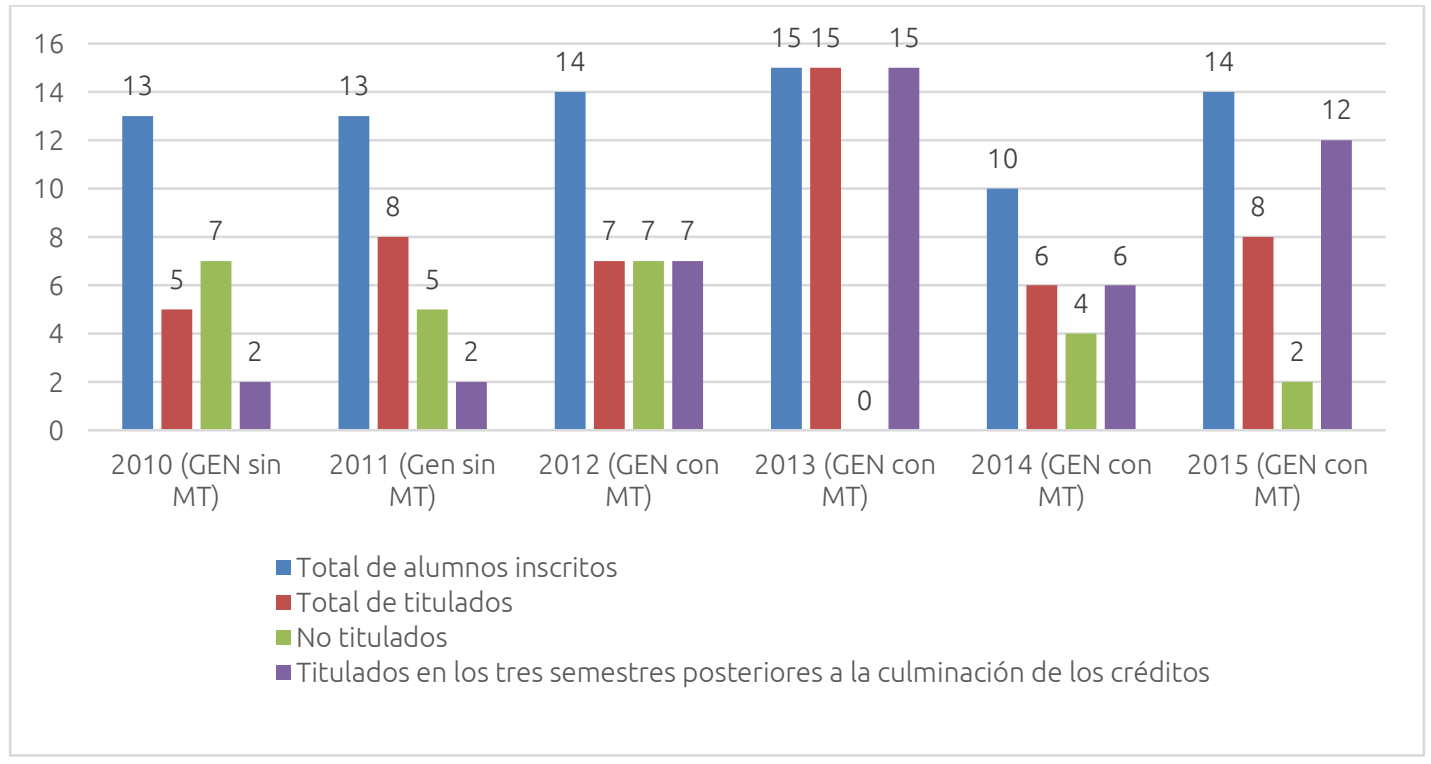

Figura 3. Titulación entre las generaciones que cursaron y no cursaron la materia con el modelo de Toulmin

De acuerdo con los datos, con el acompañamiento apoyado en el modelo de Toulmin la titulación tiende a darse en los tres semestres posteriores a la culminación de los créditos de la maestría. Es el indicador más significativo en la figura 3, obsérvese la diferencia entre los estudiantes que se titulan en 2010 y 2011 (generaciones que no estuvieron expuestas al modelo) y los que se titulan en las generaciones del 2012 al 2015. Más de $80 \%$ de los estudiantes titulados de las generaciones que no recibieron el modelo presentaron su examen tres años después de concluidos los créditos, es por eso que no se considera que haya una alta eficiencia de titulación, pues se logra años después de concluidos los créditos. Desde esta concepción no se obtiene la eficiencia real, pero observando el número de titulados de las generaciones expuestas y no expuestas al modelo, predomina un mayor número de titulados en las que fueron expuestas.

\section{Discusión y conclusiones}

En cuatro años consecutivos (de 2012 a 2016), los datos empiezan a mostrar la posible efectividad del modelo de Toulmin para la elaboración de la tesis y para la eficiencia de titulación. Respecto a 2010 y 2011, la obtención del grado comienza a presentar una tendencia sistemática al crecimiento en los tres semestres que siguen a la culminación de los créditos. Al hacer un análisis comparativo entre las generaciones que no recibieron la materia con aquellas que sí la recibieron, y que han tenido en común: 1) mismo programa educativo, 2) misma institución, 3) estudiantes seleccionados bajo procedimientos similares y 4) misma planta docente, sólo con excepción de estar expuestos a esta materia y al modelo de Toulmin, podemos inferir que ambas son variables relevantes y significativas para la eficacia de titulación.

Organizar las representaciones mentales de acuerdo con sus componentes, debatirlas en grupo y escribirlas durante el año escolar permite a los estudiantes concluir el programa con un avance de tesis difícil de abandonar. Nos es necesario aclarar que en el primer semestre se elabora el esquema y se escribe el marco teórico. En el 20. semestre se construye el método y se elabora la sección de resultados, la discusión y las conclusiones.

Se considera que la eficiencia de titulación se beneficia del modelo por las siguientes razones: 1) hay un rescate del pensamiento lógico, de aquel que busca relaciones de implicación plausibles entre nuestros razonamientos y 2) el estudiante adquiere las certezas en un inicio ausentes respecto al tema de interés. 
Formula quizás su primera inferencia, una propuesta de conocimiento y se compromete con la idea de probarla. Como experiencia se ha observado que esta primera inferencia les demanda mucho esfuerzo, se les dificulta construir una estructura gramatical afirmativa, con sujeto, predicado y verbo, en la que, de forma general, están estableciendo una posible relación causal. Parece buena estrategia disminuir la angustia ante lo intuitivo y lo tosco de las construcciones iniciales, se brinda certeza de que "así se empieza" y luego se afinará todo.

Otro elemento que brinda certeza es el debate con el grupo, la exposición a la crítica y el manejo profesional de las situaciones contra-argumentativas, que permite lo que Pereda (1996) llama "espíritu de rescate", que consiste en aprender a rescatar el valor de los aportes, incluso de aquellos que se opongan a los nuestros desde la mala fe.

El orden de las representaciones mentales facilita la ardua tarea de escribir. Hasta el momento las entregas de cada producto tienden a ser de $90 \%$ y se aprecia un gran esfuerzo por una escritura académica y auténtica. Los temas bien delimitados y el número de cuartillas con una cantidad finita y congruente con las investigaciones actuales parecen reducir la ansiedad.

Se considera también que ha sido benéfico el respeto a la propuesta de los estudiantes. No se les invita a que asuman la inferencia del asesor sino a que "afinen" su propia inferencia. El estudiante ha estado frente a los datos, por lo que su inferencia puede resultar plausible. Otro aspecto importante ha sido la lectura concienzuda de cada uno de los productos que genera el estudiante, no sólo por parte del docente sino de sus asesores.

Las entregas de los productos tienen una fecha que toma en consideración sus cargas de trabajo y otras responsabilidades de la maestría. Son, además, parte de la evaluación de la materia. Terminados los dos semestres y con un trabajo en el que las partes se unen formando el todo y agregan la portada institucional, anima a hacer lo poco que queda. Resulta para ellos inconcebible dejar "tanto trabajo" en el abandono, entonces deciden terminar la tesis.

\section{Referencias}

Barceló, A. (2012). Tipos de argumentos filosóficos. Recuperado de http://www.filosoficas.unam.mx/ abarcelo/IntroFil/Argumentos.pdf

Barrera, K. y León, R. (2012). Consideraciones metodológicas en la medición de la eficiencia terminal en la educación superior. En Z. Monroy, R. León y L. G. Álvarez (Eds.), Enseñanza de la ciencia (pp. 313-319). México: UNAM.

Carreño, A. G. (2015). La indiferenciación madre e hija como precursor de sobrepeso y atracones. Tesis de maestría, Universidad Nacional Autónoma de México, Ciudad de México.

Cleveland Clinic. (2006). El trastorno por atracón. Recuperado de www.clevelandclinic.org/health/sHIC/doc/s12249.pdf

De Gracia, M. y Castelló, A. (2002). Aproximación a los orígenes de la psicología cognitiva del pensamiento. Revista de Psicología General y Aplicada, 55(4), 515-539.

Fernández, E., Mendoza, M. A., Rodríguez, A. y López, M. A. (2013). Aspectos que inciden en la baja titulación de los alumnos de las maestrías en la Universidad Pedagógica de Durango. Praxis Investigativa ReDIE, 5(9), 6-14.

López, M., Salvo, B. y García, G. (1989). Consideraciones en torno a la titulación en las instituciones de educación superior. Revista de la Educación Superior, 18(1), 69. 
López, A., Albíter, Á. y Ramírez, L. (2008). Eficiencia terminal en la educación superior, la necesidad de un nuevo paradigma. Revista de la Educación Superior, 37(146), 135-151.

Luna, B. (2011, noviembre). Factores de la influencia en la gestión de la planeación académica para el logro de la eficiencia terminal del estudiante de educación superior. Documento presentado en el XI Congreso Nacional de Investigación Educativa, Ciudad de México. Recuperado de http://www.comie.org.mx/congreso/memoriaelectronica/v11/docs/area 13/1420.pdf

Medina-Otero, T. (2011). Titulación: estrategias y acuerdos epistemológicos. Investigación universitaria multidisciplinaria, 8, 26-33.

Pereda, C. (1994). Vértigos argumentales. España: Anthropos.

Perelman, C. y Olbrecht, L. (2006). Tratado de la argumentación. La nueva Retórica. España: Gredos.

Pérez, M. P. (1990). Psicología del razonamiento probabilístico. Madrid: Universidad Autónoma de Madrid.

Toulmin, S. (1958). The uses of argument. Cambridge University Press.

Universidad Nacional Autónoma de México. (2015). Agenda Estadística unAM. Recuperado de http://www.planeacion.unam.mx/Agenda/2015/disco/

Van Eemeren, F. H. y Grootendorst, R. (2004). A systematic theory of argumentation. The pragma-dialectical approach. Cambridge University Press.

Villagra, M. y Casas, M. (2010, mayo). La falta de tiempo docente y otros avatares. Textos y pretextos que dificultan la elaboración de la tesis. Documento presentado en el I Congreso Argentino y Latinoamericano de Posgrados en Educación Superior, San Luis, Argentina. 